EPJ Web of Conferences 35, 05006 (2012)

DOI: $10.1051 /$ epjconf/20123505006

(C) Owned by the authors, published by EDP Sciences, 2012

\title{
Applications of a 6.5T Superconducting Solenoidal Separator
}

\author{
D.J. Hinde ${ }^{\mathrm{a}}$, M. Dasgupta, M.D. Rodriguez ${ }^{\mathrm{b}}$, R. Rafiei ${ }^{\mathrm{c}}$, M.L. Brown ${ }^{\text {d }}$, A.J. Horsley, I.P. Carter, T. Kibedi, N. Lobanov, \\ D.C. Weisser, M. Evers, G.J. Lane, D.H. Luong, A. Wakhle, and E. Williams \\ Department of Nuclear Physics, RSPE, Australian National University, ACT 0200, Australia
}

\begin{abstract}
A 6.5 Tesla superconducting gas-filled solenoid (SOLITAIRE) has been developed at the Heavy Ion Accelerator Facility at the ANU as a reaction product separator. Key features of the device allowing its application for precise measurement of heavy ion fusion cross sections are described. The physical separation of beam particles and the high efficiency $(\sim 80 \%)$ transport of heavy ion fusion products open up applications in nuclear structure physics, and in materials science. Finally, the developments to allow its application to providing beams of light radioactive isotopes (SOLEROO) are described.
\end{abstract}

\section{Introduction}

Nuclear fusion and fission are arguably the most important and complex nuclear reactions, which provide energy both in the cosmos and on Earth. In the last 20 years, our understanding of the fusion of heavy nuclei has undergone a revolution, with the influence of the identity and properties of the colliding nuclei now understood to play a very important role. Research at the Australian National University has played a significant part in this.

The first comprehensive measurements of fusion barrier distributions showed directly the quantum eigenstates at the barrier [1]. Their influence on the subsequent dynamics in reactions forming heavy elements was demonstrated [2], as was the importance of mass-asymmetry of the colliding nuclei [3]. In collisions of lighter nuclei such as ${ }^{6,7} \mathrm{Li}$, suppression of complete fusion by breakup was experimentally demonstrated [4] for the first time. More recently the importance of transfer reactions in triggering breakup was demonstrated [5], where a change in the identity of the light nucleus can make a large difference to the outcome. Finally, the roles of dissipation and quantum decoherence are now being investigated [6] as mechanisms to explain significant deviation of fusion cross sections from the most sophisticated coherent coupled channels quantum calculations [7]. In these experiments, the development of instrumentation to enable precise absolute cross section measurements has played an important role. In this presentation the development and applications of a new large solid angle fusion product separator [8] is described.

Because of the high momentum of heavy ion beams, the products of nuclear reactions are often focused around the beam direction. In particular, nuclear fusion products carry all the beam momentum. They are deflected from the

\footnotetext{
a e-mail: david.hinde@anu.edu.au

b Current address: Department of Electronic Materials Engineering, RSPE, ANU, ACT 0200, Australia

c Current address: Australian Nuclear Science and Technology Organisation, Lucas Heights, NSW 2232, Australia

d Current address: Navman NZ Ltd., 7-11 Kawana St, Northcote, Auckland, New Zealand
}

beam direction $\left(0^{\circ}\right)$ by the generally much smaller recoil momentum imparted by evaporated light particles, emitted as they cool to form evaporation residues (ERs). For thick targets, angular scattering in the target can also spread the angles significantly. Nevertheless, fusion products are generally found inside a $10^{\circ}$ cone centred around $0^{\circ}$.

To measure fusion cross sections, it is very desirable to physically separate the ERs from the beam particles before attempting detection, as the intensity of the Rutherfordscattered beam is typically at best $10^{4}$ times that of the ERs, and can be more than $10^{12}$ times larger, while the direct beam is many orders of magnitude larger still. Thus any detector will be overwhelmed by the rate of beam particles. A velocity filter can separate the slow ERs from beam particles, but a large solid angle is problematic. In a magnetic field alone (where the bending radius depends only on the ratio of momentum to charge) ERs and beam particles can only be separated if their charge states are different, since on average both have the same momentum. Since each will have a distribution of charge states from the target, obtaining good separation may be impossible.

A gas-filled solenoid promises to be the ideal solution. In a solenoidal field, in simple terms, the radial component of the particle velocity interacts with the axial component of the field, bending trajectories back to the beam (solenoid) axis at different focal lengths, depending on the charge state. Due to the azimuthal symmetry, a solenoid can provide a large solid angle. With a gas filling such that there are a sufficient number of charge-changing collisions, particle trajectories will correspond to that of the mean charge state in the gas. Finally, in He gas, generally the ERs have a lower charge state than the beam particles. Only trajectories close to the axis (with negligible radial velocity component) undergo no deflection or separation, and have to be blocked. The only remaining challenge is to achieve sufficient bending power to re-focus the ERs from their initially diverging trajectories leaving the target.

At the Heavy Ion Accelerator Facility in the Department of Nuclear Physics at the ANU, we have developed a 6.5 Tesla superconducting solenoidal separator, called SOLITAIRE [8]. In gas-filled mode, it has been used to measure precise heavy-ion fusion cross-sections, and to 


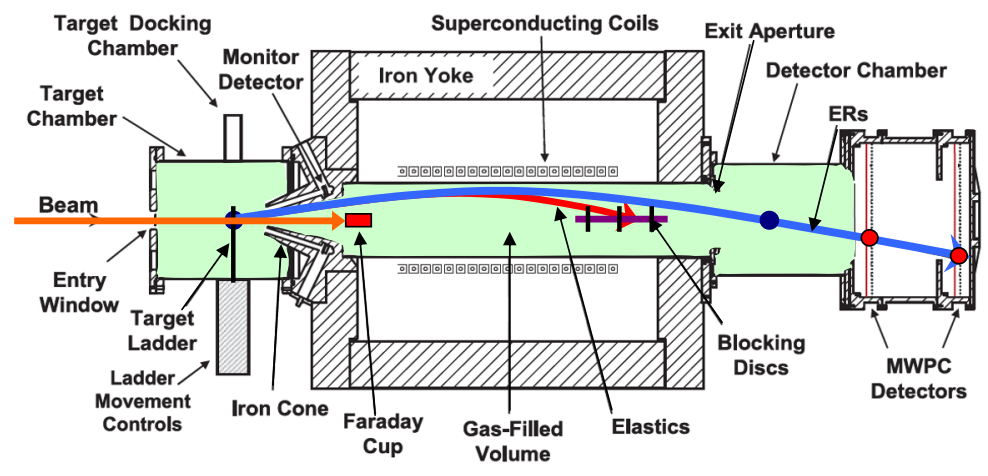

Fig. 1. Schematic diagram of the components of the solenoidal separator. When used with gas filling, the He gas region is shown by the light green shading. The points where the ER trajectory is closest to the axis are shown by blue circles, and the interaction points with the two MWPC tracking detectors by red circles. The helical trajectories of elastically scattered beam particles (red) and ERs (blue) are represented by the radial separation from the axis. More details can be found in Ref. [8].

make measurements of $\gamma$-rays for nuclear structure research. It has also been used to recoil-implant short-lived radioactive probe nuclei into materials for atomic-scale studies of the host lattice via the hyperfine interactions with the probe. In gas-free mode, as SOLEROO, we have demonstrated $[9,10]$ its application to produce light radioactive ion beams e.g. ${ }^{6} \mathrm{He}$.

\section{Description}

A diagram of the SOLITAIRE configuration [8] is shown in figure 1 . The solenoid consists of a $60 \mathrm{~cm}$ long coil, in a cryostat with a $20.5 \mathrm{~cm}$ clear bore. The cryostat is located between low-carbon 1010 steel discs, connected by eight steel bars. This steel yoke, weighing $2500 \mathrm{~kg}$, together with the coil, makes up a magnetic circuit, minimizing the escape of the magnetic field into the surroundings. A $20 \mathrm{~cm}$ diameter vacuum pipe passing through penetrations in the steel discs, and through the bore, connects the target and detector chambers, which are upstream and downstream respectively of the solenoid. At the entrance of the target chamber is a cassette of 6 interchangeable carbon entrance foils, $\sim 25 \mu \mathrm{g} \mathrm{cm}^{-2}$ in thickness, which separate the He gas from the beam tubes and accelerator vacuum. Viewing the target at $18^{\circ}$ are four Si monitor detectors, for normalization of cross sections. At the end of the detector chamber are two multi-wire proportional counters (MWPCs), which each give an energy loss and time signal, together with $\mathrm{X}$ and $\mathrm{Y}$ position signals. They allow the identification of ERs, and tracking of their trajectories.

Examples of MWPC position spectra are given in figure 2. Here images of a mask at the entrance to the solenoid (shown as an inset in the upper panel) are presented, for elastically scattered ${ }^{58} \mathrm{Ni}$ ions. Data are shown for two magnetic field values, without any gas filling the bore. The excellent position resolution is apparent. Each repeat of the hole pattern, at a different radius, corresponds to a different charge state of the beam particles. Since the focal point is already in front of the detectors at the lower field setting, increasing the field moves the images radially outwards.
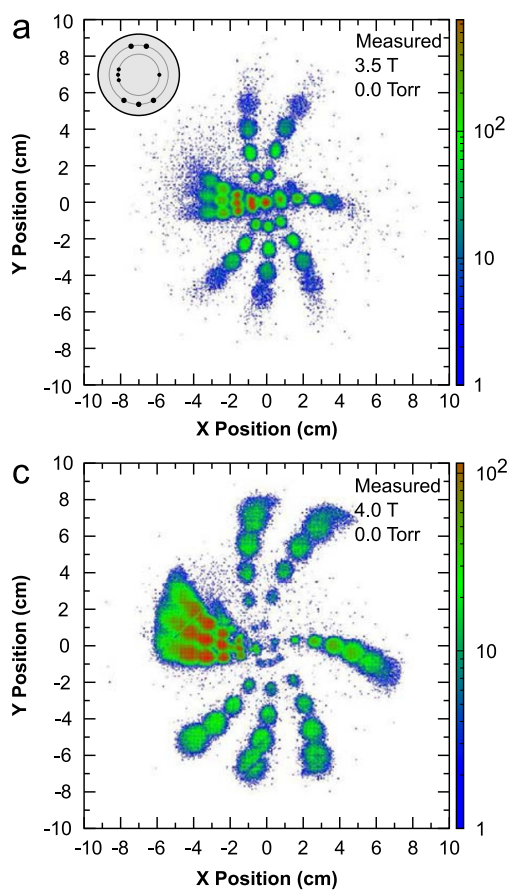

Fig. 2. Images at the MWPC detector of elastically scattered 220 $\mathrm{MeV}{ }^{58} \mathrm{Ni}$ projectiles at different magnetic fields with no gas. The collimator mask pattern is shown in the upper panel, which is mirrored in the position spectrum for each charge state.

\section{Evaporation residue cross sections}

Using a pulsed beam, ERs can be clearly identified [8] from the time and energy loss signal in one or both of the MWPC detectors. Figure 3 shows the position spectrum for ERs, without the entrance mask, and with 1 Torr of He gas filling the solenoid bore. The suppression of yield in the centre of the image is due to the axial Faraday cup and elastic blocking discs (see figure 1). As with the image of the mask, the radial distribution measured in the MWPCs reflects the angular distribution of the ERs leaving the target, the average charge state, and trajectory fluctuations.

To determine ER cross sections, the efficiency of SOLITAIRE must be determined. A computer simulation [12] of the device, and of the ion trajectories in gas, has 


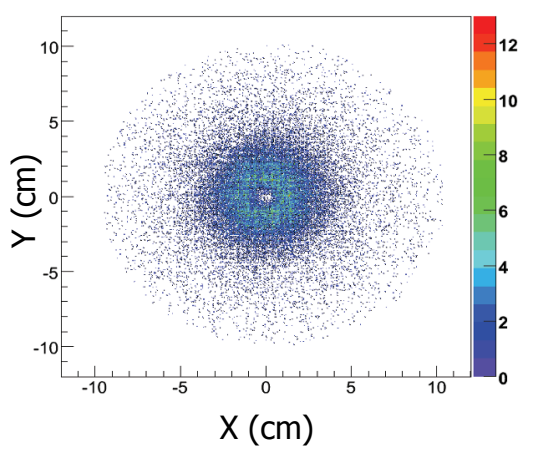

Fig. 3. Position spectrum of ERs on the MWPC detector.

been developed to calculate losses of ERs through SOLITAIRE. Calculation of the efficiency requires knowledge of both the effective average charge state of the ERs, and the initial ER angular distribution from the target. If the former is determined, the latter can be inferred from the radial distribution. In addition, a novel method to determine the shape of the ER angular distribution from the time-offlight spectrum alone is described in the contribution to this Symposium of I.P. Carter et al. [11].

The effective gas charge state can be determined by "tuning" the mean charge state in the simulation to give the minimum image size [8] at the MWPC at the same field as found experimentally. A second method, which does not require scanning the magnetic field, makes use of the measurements of ER position in the two MWPC detectors, which are separated axially by $24 \mathrm{~cm}$. This approach is illustrated in figure 4, which shows axial distance (Z) upstream from the back MWPC, and distance from that axis (X). The two purple lines at the bottom indicate the positions of the two MWPCs. Two example trajectories are indicated by the black and grey lines, extrapolated from the measured interaction points on each detector (circles). A density plot of actual ER trajectories determined by this method is shown in the background. Determining the axial distance of least confusion (focal point), and matching that in the simulation by varying the average charge state used, allows the effective charge state to be reliably determined, and thus the trajectories and transmission efficiencies. This method relies on minimal deviation of the trajectories from

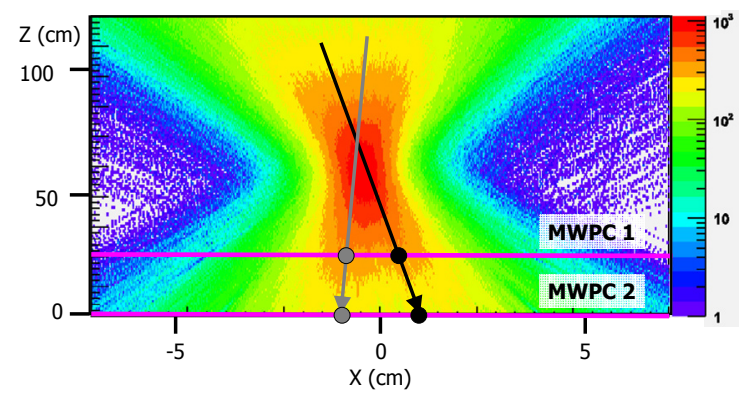

Fig. 4. Diagram illustrating the extrapolation of trajectories to determine the effective charge state. The detectors are at the bottom (purple lines) and illustrative interaction points (circles) of two trajectories define the ion path. The background image shows experimental ER trajectories, which indicate a slight axial misalignment of the beam with respect to the solenoid axis. straight lines between the detectors and the focal point. The simulation shows that this approximation is valid since in an experiment the focus should be set about half way between elastic blocking discs (at the solenoid exit) and the first MWPC detector. Scattering of the ERs in the foils of the first MWPC results in a larger deduced image size in X than when measured directly by adjusting the field to place the image at the MWPC plane. This does not affect the determination of the position of the focus in Z. Detailed ER cross section excitation functions have been measured for fusion of ${ }^{58} \mathrm{Ni}$ with ${ }^{58,60,64} \mathrm{Ni}$, allowing high quality fusion barrier distributions [1] to be determined [8].

\section{Evaporation residue $\gamma$-ray decay studies}

The efficient transport of evaporation residues to a location with a much reduced flux of beam particles is beneficial for applications based on measurements of radiation emitted in the decay of the ERs.

Nuclear structure studies of decay from isomeric states can benefit from a reduced $\gamma$-ray background. An advantage of SOLITAIRE is the shorter flight path to the focus, and larger solid angle than other separators, allowing studies of short-lived isomers (down to 100ns). A $\gamma$ spectroscopy module SOLENO-GAM has been developed at the ANU [13], which replaces the MWPC module behind the solenoid, and has enabled characterization of an isomer in ${ }^{189} \mathrm{~Pb}$ [14].

Another application is in condensed matter physics. Short-lived radioisotopes are used to probe materials with hyperfine interactions techniques such as TimeDifferential Perturbed Angular Correlation [15]. Here fusion products are implanted into a material, and in their subsequent decay, the angular correlation in a $\gamma$-ray cascade is sensitive to both the internal magnetic field and the electric field gradient at the probe location. SOLITAIRE eliminates the undesirable high flux of beam particles. Recent measurements showed good transmission efficiency for ${ }^{100} \mathrm{Pd}$ ions, even when formed in fusion of a projectile as light as ${ }^{12} \mathrm{C}$ bombarding a ${ }^{92} \mathrm{Zr}$ target, and the expected TDPAC ratio function was observed in an existing TDPAC setup following implantation of ${ }^{100} \mathrm{Pd}$ into a $\mathrm{Zn}$ host, opening up potential spintronics research applications.

\section{Producing light radioactive beams}

Radioactive nuclei far from stability are expected to have different properties from stable nuclei. In particular, many close to the neutron and proton drip-lines show neutron or proton halos - neutron or proton wave-functions that extend far from the core because of the low binding energy [16]. The interactions of halo nuclei may show very different behaviour from well-bound nuclei. It has been suggested that irreversible interactions of the halo nucleon(s) with those in the target nucleus [17] may be important, even at large separations, in contrast with normal expectations for well-bound nuclei. Experiments are difficult, and even measurements of the dominant fusion process require careful analysis [18], and have not yet conclusively shown the major differences from fusion of stable nuclei that might have been expected. Further systematic 


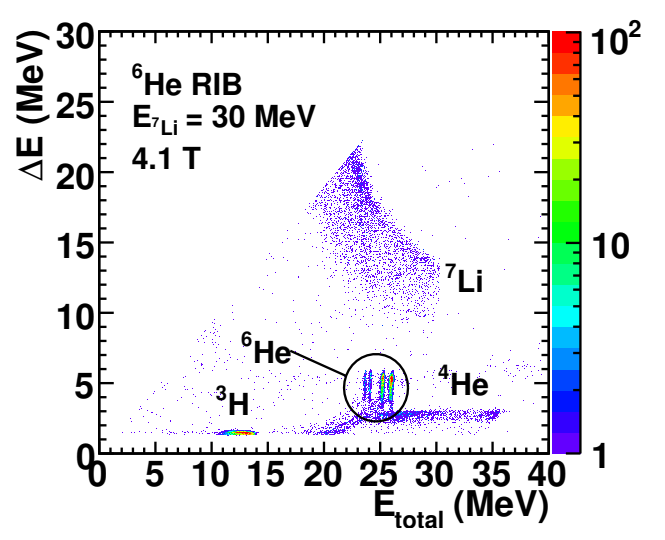

Fig. 5. Energy loss vs. total energy of particles reaching the secondary target position for ${ }^{7} \mathrm{Li}$ bombarding a thin target of ${ }^{9} \mathrm{Be}$ at the optimal field for ${ }^{6} \mathrm{He}$, at which nearly $50 \%$ of the particles comprise ${ }^{6} \mathrm{He}$.

measurements of both fusion and breakup, and the interaction between the two, are still required.

The pioneering TwinSol facility at the University of Notre Dame $[19,20]$, and the later RIBRAS facility at the University of Sao Paulo [21] have shown that superconducting solenoids can be effectively used to produce secondary beams of light radioactive nuclei through nucleon transfer reactions in-flight. For light nuclei, only one (or at most two) charge states are likely to be populated, so no gas filling of the solenoid bore is used.

The application of SOLITAIRE to RIB production at the ANU (SOLEROO) has been described in Refs. [9, 10]. A key quantity in precision measurements is beam purity and energy definition. To address these, two thin gas proportional detectors are located after the solenoid, but before the secondary target. These are capable of sustaining count-rates over $10^{6} / \mathrm{sec}$. Triggered by a signal in one of the detectors viewing the secondary target, the PPACs record the time, energy loss and position of the ion preceding the trigger. This allows identification of the charge and mass of the beam particle, and reconstruction of the trajectory. The trajectory allows correction for the incidence angle and interaction point of the RIB particle on the target, and also improves the energy resolution by measurement of the focal length. This tagged beam method is practical if the total rate in the tracking detectors is not too high.

To investigate the impurities in the RIB, a silicon $\Delta \mathrm{E}$ E detector telescope has been placed at the position of the secondary target, and the particles detected are shown in figure 5 . The best ${ }^{6} \mathrm{He}$ purity was found to be $\sim 50 \%$ for the standard [19] reaction ${ }^{9} \mathrm{Be}\left({ }^{7} \mathrm{Li},{ }^{6} \mathrm{He}\right){ }^{10} \mathrm{~B}$. This is a good result, since for a RIB rate of $10^{5} / \mathrm{sec}$, a purity level of $10 \%$ would be sufficient. Other targets were investigated, as shown in figure 6. Results from a ${ }^{\text {nat }} \mathrm{SiO}_{2}$ target suggest ${ }^{29,30} \mathrm{Si}$ may be better targets than ${ }^{9} \mathrm{Be}$. This needs confirmation with enriched targets.

With an optimised geometry of blocking discs and apertures determined from these measurements and simulations, the next step is to make nuclear reaction measurements with the ${ }^{6} \mathrm{He}$ radioactive beam.

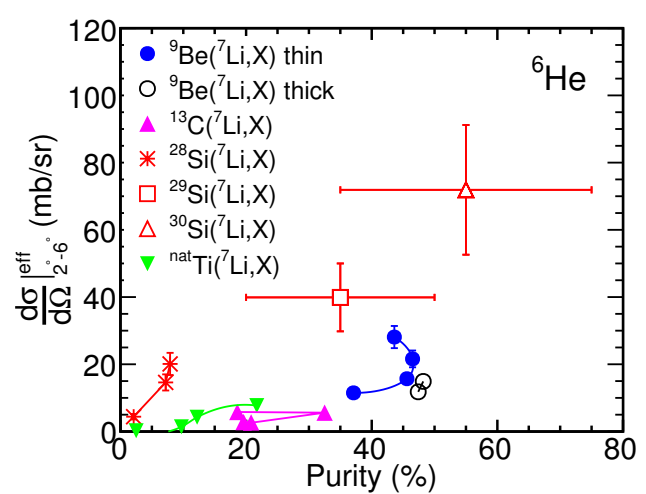

Fig. 6. Graph of beam purity and reaction cross section for ${ }^{7} \mathrm{Li}$ beams bombarding various targets, at energies ranging from 20 to $35 \mathrm{MeV}$. The lowest energy gives the lowest yield. The measurements for ${ }^{29,30} \mathrm{Si}$ derive from a target of ${ }^{\text {nat }} \mathrm{SiO}_{2}$.

\section{References}

1. J.X. Wei et al., Phys. Rev. Lett. 67, (1991) 3368; J.R. Leigh et al., Phys. Rev. C52, (1995) 3151; M. Dasgupta et al., Annu. Rev. Nucl. Part. Sci. 48, (1998) 401

2. D.J. Hinde et al., Phys. Rev. C53, (1996) 1290; J.C. Mein et al., Phys. Rev. C55, (1997) R995, D.J. Hinde et al., Phys. Rev. Lett. 100, (2008) 202701

3. A.C. Berriman et al., Nature 413, (2001) 144; D.J. Hinde et al., Phys. Rev. Lett. 89, (2002) 282701; D.J. Hinde and M. Dasgupta, Nucl. Phys. A787, (2007) 176c

4. M. Dasgupta et al., Phys. Rev. Lett. 82, (1999) 1395; M. Dasgupta et al., Phys. Rev. C66, (2002) 041602

5. R. Rafiei et al., Phys. Rev. C81, (2010) 024601; D.H. Luong et al., Phys. Lett. B695, (2011) 105

6. M. Dasgupta et al., Phys. Rev. Lett. 99, (2007) 092701; M. Dasgupta et al., Nucl. Phys. A787, (2007) 144c; A. Diaz-Torres et al., Phys. Rev. C78, (2008) 064604; D.J. Hinde et al., Nucl. Phys. A834, (2007) 117c; M. Evers et al., Phys. Rev. C84, (2011) 054614

7. K. Hagino, N. Rowley, and A. T. Kruppa, Comput. Phys. Commun. 123, (1999) 143

8. M.D. Rodriguez et al., Nucl. Instr. Meth. in Phys. Res. A614, (2010) 119

9. R. Rafiei et al., Nucl. Instr. Meth. in Phys. Res. A631, (2011) 12

10. A.J. Horsley et al., Nucl. Instr. Meth. in Phys. Res. A646, (2011) 174

11. I.P. Carter et al., EPJ Web of Conf. this volume (2012)

12. M.L. Brown, M.Sc. thesis (Australian National University 2012)

13. P. Nieminen et al., AIP Conf. Proc. 831, (2006) 517

14. G.D. Dracoulis et al., Phys. Rev. C79, (2009) 031302

15. A. Abiona et al., EPJ Web of Conf. this volume (2012)

16. I. Tanihata et al., Phys. Lett. B160, (1985) 380

17. D.J. Hinde and M. Dasgupta, Nature 431, (2004) 748 and ref. therein

18. D.J. Hinde et al., Phys. Rev. C81, (2010) 064611

19. F. D. Becchetti et al., Nucl. Instr. Meth. B56-7, (1991) 554

20. F. D. Becchetti et al., Nucl. Instr. Meth. A505, (2003) 377

21. R. Lichtenthaler et al., Eur. Phys. J. A25, (2005) 733 